\title{
The distinction blurs between an autoimmune versus microbial hypothesis in multiple sclerosis
}

\author{
David A. Hafler \\ Harvard Medical School and Brigham and Women's Hospital, Harvard Institutes of Medicine, 77 Avenue Louis Pasteur, \\ Boston, Massachusetts 02115, USA. E-mail: hafler@cnd.bwh.harvard.edu.
}

Multiple sclerosis (MS) is an inflammatory disease limited to the central nervous system (CNS) white matter. The CNS inflammation varies among different patients, and consists of variable degrees of $\mathrm{T}$ lymphocytes, macrophages, B lymphocytes, and antibodies at the leading edge of the white matter destruction (1-3). Among the fundamental questions yet to be answered in understanding the pathophysiology of MS, and for that matter, other autoimmune diseases, are (a) what is the antigenic target driving the inflammation in the target organ, and (b) what initiates the inflammation? There were thought to be 2 possibilities regarding the initiating event in MS. The first is that the CNS white matter is structurally normal, and that an autoimmune response initiated by autoreactive $\mathrm{T}$ cells, as shown possible by the experimental autoimmune encephalomyelitis (EAE) model, mediates the initial inflammatory insult. The second possibility is that the inflammation is a result of some alteration of the CNS white matter, which could be the result of a microbial CNS infection. The elucidation of the mechanism responsible for the initiation of MS has obvious implications for the further development of specific therapies. However, this distinction between an autoimmune hypothesis, where the CNS white matter is normal, and the microbial hypothesis, where there is an infected CNS with regard to the etiology of MS, may be more blurred then previously thought.

One of the more important discoveries over the past decade in understanding the pathogenesis of MS is the concept of epitope spreading. In this scenario, first demonstrated by Sercarz and colleagues, it was shown that the inflammatory process initiated by $\mathrm{T}$ cell recognition of 1 myelin protein epitope subsequently leads to the activation of autoreactive $\mathrm{T}$ cells recognizing other epitopes of the same protein (4). In time, there is activation of T cells recognizing other myelin proteins that presumably get degraded and then loaded into the MHC of local antigenpresenting cells (APCs). The discovery of epitope spreading was important for the investigation of MS, because it suggested that the answer to the first fundamental question - what is the antigenic target driving the disease? - was unlikely to be found in the discovery of a single antigen. By the time a patient with the disease and recurrent attacks is seen in the clinic, there is likely to be activation of myelin-reactive $T$ cells recognizing many myelin epitopes and proteins. These experiments in the EAE model may explain data from our laboratory showing myelin-reactive $T$ cells activated against both myelin basic protein and proteolipid protein in the same MS patient (5).

What, then, is the initiating event leading to epitope spreading in patients with MS? The report by Katz-Levy et al. in this issue of the JCI provides new mechanistic insight into the understanding of how viruses can induce human autoimmune diseases (6). Here again, the investigation of the human disease is aided by the experimental animal. Theiler's murine encephalomyelitis virus-induced demyelinating disease (TMEV-IDD) is caused by direct
CNS infection by the virus, leading to TMEV-specific CD 4 cells that cross into the CNS and target virally infected CNS-resident APCs (reviewed in ref. 7); depletion or tolerance of T lymphocytes abrogates the disease (8). It was recently shown by Miller et al. that whereas the initial T-cell response is directed to TMEV, the chronic T cell-mediated disease involved the activation of myelinreactive CD4 cells (9). However, the mechanism leading to the activation of naive myelin-reactive CD4 cells was not clear. Specifically, a widely held belief in the pathogenesis of autoimmune disease is that some form of molecule mimicry induces the initial activation of myelin-reactive T cells (10), followed by epitope spreading and recognition of multiple CNS myelin proteins (4). In this report by Katz-Levy et al., it is shown that microglia (local CNS APCs) isolated directly from the CNS of TMEV-infected mice were able to endogenously present epitopes of a myelin antigen, proteolipid protein, to T-cell lines. These experiments demonstrate that with viral infection of the CNS, myelin antigens can be processed and presented by microglial cells in such a way as to activate naive T cells. These findings raise the question as

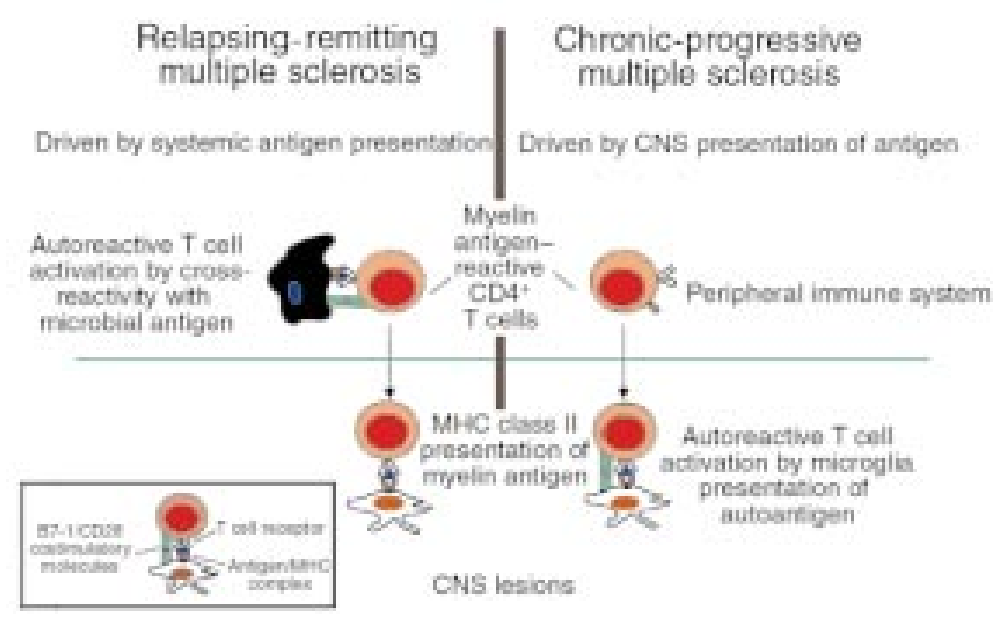

Figure 1

Pathogenesis of autoimmune-mediated inflammatory CNS disease. 


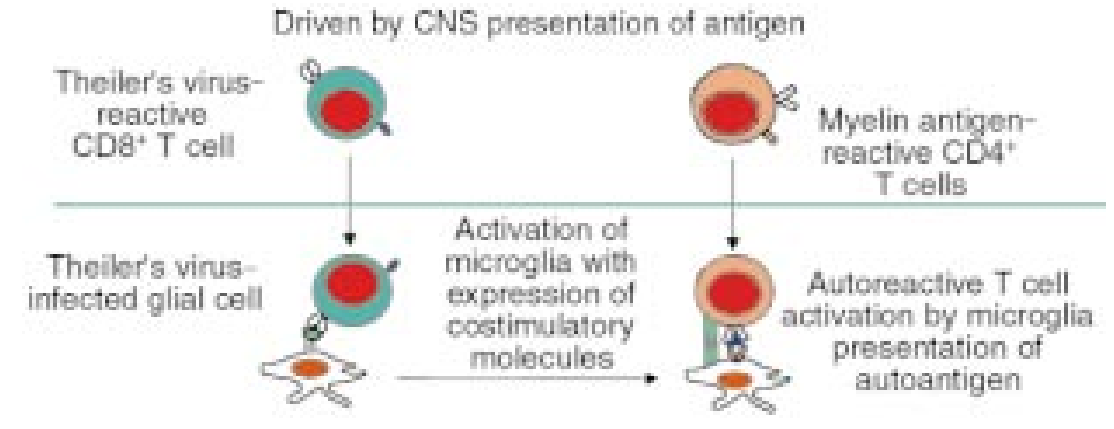

Figure 2

Pathogenesis of viral-induced autoimmune inflammatory CNS disease.

to why epitope spreading does not occur in other forms of CNS inflammation such as stroke, where there is massive tissue destruction and inflammation by macrophages upon activation of microglial cells. The explanation for this may relate to a series of observations made by Miller et al., who demonstrated that expression of B7-1 on local APCs is critical for the induction of epitope spreading in the relapsing SJL EAE model (11). In patients with MS, perhaps similar to what is observed in EAE, there is significant B71 expression in the CNS inflammation found in MS plaques (12-14). In marked contrast, the inflammation associated with stroke consists almost entirely of B7-2 expression (14). In addition, with chronic viral infection such as subacute sclerosing panencephalitis, there is also B7-1 expression in the CNS white matter (14). Thus, either viral infection or infiltration of activated myelin-reactive Th1 T cells in the CNS is accompanied by B7-1 expression, leading to myelin breakdown that may then lead to epitope spreading and chronic CNS inflammation.

MS can be broadly divided into 2 forms of disease: the early relapsingremitting type, where there is an average of 1.5 attacks per year clinically and 10 new lesions a year by magnetic resonance imaging (MRI); and chronic progressive disease, where, in marked contrast to patients with relapsingremitting disease, there is a slowly progressive, downhill course without clinical exacerbations. Patients with chronicprogressive MS tend to have fewer new MRI lesions, as measured by dye leaking through the blood vessels (gadolinium enhancement), an indication of acute inflammation (15). Approximately one third of patients with relapsing-remitting disease go on to progressive disease, and approximately $10 \%$ of MS patients begin with progressive disease without any previous relapsing-remitting events (primary-progressive MS). What are the implications of these findings in TMEV CNS infection with MS? The animal models can perhaps provide insight into the group of syndromes that we call MS. Specifically, these experimental models lead us to hypothesize that early relapsing-remitting MS may be similar to the EAE model where there is activation of autoreactive T cells mediated by peripheral immune system stimulation by microbial antigens cross-reactive with CNS myelin antigens (Figure 1). This is consistent with the only known trigger associated with MS: antecedent viral infections (16). Thus, the myriad clinical and subclinical viral and bacterial infections we experience every year result in the specific and nonspecific activation of the immune system that can induce the activation of autoreactive $T$ cells (17). In contrast, it can be hypothesized that in the transition to chronic-progressive MS, as seen in the TMEV model, the inflammation is driven by chronic CNS presentation of self antigen (Figures 1 and 2). In this model, the environmental influences of repeated microbial infections are not necessary for the peripheral activation of the immune system; instead, the CNS becomes a depot of myelin antigens presented by activated APCs expressing B71. This steady state is predicted to be associated with the slow, insidious progression of disease. The results presented by Katz-Levy et al. now tie together the autoimmune and viral models of $\mathrm{T}$ cell-mediated CNS damage, by demonstrating that viral infection of the CNS can directly lead to epitope spreading by microglia presentation of self antigen in chronic autoimmune disease (Figure 2). This might explain why the insidious, secondary-progressive MS that follows repeated exacerbations may clinically and mechanistically resemble chronic TMEV infection.

Is there evidence that a pathogen can chronically infect the CNS in humans, leading to a slowly progressive demyelinating disease similar to the model proposed by TMEV CNS infection in mice? The answer is yes: HTLV-I-associated myelopathy/tropical spastic paraparesis (HAM/TSP) is a well-defined human disease that may be closely modeled by TMEV (Figure 3) (18). As with TMEV, there is infection of the CNS, albeit with qualitatively lower amounts of HTLV-I virus in CNS glial cells. In addition, there is a very high frequency of CD8 T cells recognizing the virus in the circulation $(19,20)$, which has recently been well

\section{Theiler's murine encephalomyelitis virus}

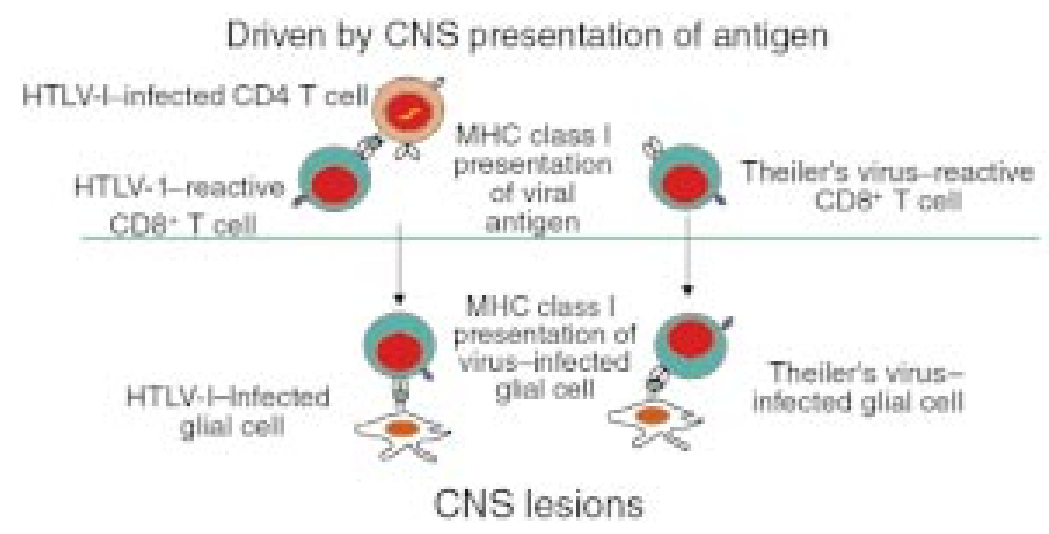

Figure 3

Pathogenesis of immune-mediated inflammatory CNS viral disease. 
enumerated by tetramer staining of the cell population (21). Thus, HAM/TSP is a chronic CNS infection with a high frequency of antigen-reactive $T$ cells associated pathologically with inflammatory demyelinating disease. As seen clinically in primary-progressive MS, there is a slowly progressive spastic paraparesis accompanied frequently by MRI lesions, increased cerebrospinal fluid IgG levels, and oligoclonal banding (18). It could be argued that HAM/TSP is part of the MS syndrome, with a clearly defined pathogen. While we can easily discern MS from HAM/TSP by the diseases' predilections for different genetic hosts and the high titers to HTLV-I, this disease could fit into the syndrome of primary-progressive MS. It is unknown whether HAM/TSP has an autoimmune component; however, the investigations reported here suggest the importance of this issue.

In summary, I suggest that (a) MS is a syndrome of diseases, some induced by viral infections of the CNS, and others by direct autoimmune infiltration of the CNS; (b) the progressive forms of the disease are driven by chronic CNS presentation of endogenous self antigen resulting from epitope spreading, whereas the relapsing-remitting forms of the disease are driven by exogenous environmental stimuli, such as infections with microbes cross-reactive with self antigens; (c) CNS viral infection or repeated episodes of myelin-reactive $\mathrm{T}$ cell activation in the systemic circulation leads to presentation of myelin antigen by B7-1-expressing microglial cells and to chronic-progressive forms of autoimmune demyelinating disease; and (d) HAM/TSP is a form of primaryprogressive MS induced by viral infection of the CNS. Thus, the demonstration of activated autoreactive $T$ cells in MS patients $(22,23)$ is consistent with a role of myelin-autoreactive $T$ cells in the pathogenesis of disease, whether the disease is mediated by viral or autoimmune etiologies. For now, the distinction blurs even further between the microbial versus autoimmune hypothesis for MS.

1. Ozawa, K., et al. 1994. Patterns of oligodendroglia pathology in multiple sclerosis. Brain. 117:1311-1322.

2. Lassmann, H., Raine, C.S., Antel, J., and Prineas, J.W. 1998. Immunopathology of multiple sclerosis: report on an international meeting held at the Institute of Neurology of the University of Vienna. J. Neuroimmunol. 86:213-217.

3. Lucchinetti, C.F., Bruck, W., Rodriguez, M., and Lassmann, H. 1996. Distinct patterns of multiple sclerosis pathology indicates heterogeneity on pathogenesis. Brain Pathol. 6:259-274.

4. Lehmann, P.V., Forsthuber, T., Miller, A., and Sercarz, E.E. 1992. Spreading of T-cell autoimmunity to cryptic determinants of an autoantigen. Nature. 558:155-157.

5. Zhang, J., Markovic, S., Raus, J., Weiner, H.L., and Hafler, D.A. 1994. Increased frequency of IL-2 responsive $T$ cells specific for myelin basic protein and proteolipid protein in peripheral blood and cerebrospinal fluid of patients with multiple sclerosis. J. Exp. Med. 179:973-984.

6. Katz-Levy, Y., et al. 1999. Endogenous presentation of self myelin epitopes by CNS-resident APCs in Theiler's virus-infected mice. J. Clin. Invest. 104:599-610

7. Miller, S.D., and Gerety, S.J. 1990. Immunologic aspects of Theiler's murine encephalomyelitis virus (TMEV)-induced demyelinating disease. Semin. Virol. 1:263-272.

8. Karpus, W., Pope, J., Peterson, J., Dal Canto, M. and Miller, S. 1995. Inhibition of Theiler's virusmediated demyelination by peripheral immune tolerance induction. J. Immunol. 155:947-957.

9. Miller, S.D., et al. 1997. Persistent infection with Theiler's virus leads to CNS autoimmunity via epitope spreading. Nat. Med. 3:1133-1136.

10. Fujinami, R.S., and Oldstone, M.B. 1985. Amino acid homology between the encephalitogenic site of myelin basic protein and virus: mechanism for autoimmunity. Science. 230:1043-1045.

11. Miller, S.D., et al. 1995. Blockage of CD28/B7-1 interaction prevents epitope spreading and clinical relapses of murine EAE. Immunity. 3:739-745.

12. De Simone, R., et al. 1995. The costimulatory molecule B7 is expressed on human microglia in culture and in multiple sclerosis lesions. J. Neuropathol. Exp. Neurol. 54:175-187.

13. Williams, K., Ulvestad, E., and Antel, J.P. 1994. B7/BB-1 antigen expression on adult human microglia studied in vitro and in situ. Eur. J. Immunol. 24:3031-3037.

14. Windhagen, A., et al. 1995. Expression of costimulatory molecules B7-1 (CD80), B7-2 (CD86), and interleukin 12 cytokine in multiple sclerosis lesions. J. Exp. Med. 182:1985-1996.

15. Paty, D.W., and Li, D.K.B. 1993. Interferon beta$1 \mathrm{~b}$ is effective in relapsing-remitting multiple sclerosis. II. MRI results of a multicenter, randomized, double-blind, placebo-controlled trial UBC MS/MRI Study Group and the IFNB Multiple Sclerosis Study Group. Neurology. 43:662-667.

16. Sibley, W.A., Bamford, C.R., and Clark, K. 1985 Clinical viral infections and multiple sclerosis. Lancet. 1:1313-1315

17. Brod, S.A., Purvee, M., Benjamin, D., and Hafler, D.A. 1990. T-T cell interactions are mediated by adhesion molecules. Eur. J. Immunol. 20:2259-2268.

18. Höllsberg, P., and Hafler, D.A. 1993. Pathogenesis of diseases induced by human lymphotropic virus type I infection. N. Engl. J. Med. 328:1173-1182.

19. Jacobson, S., Raine, C.S., Mingioli, E.S., and McFarlin, D.E. 1988. Isolation of an HTLV-I-like retrovirus from patients with tropical spastic paraparesis. Nature. 331:540-543.

20. Jacobson, S., et al. 1990. Circulating CD8+ cytotoxic T lymphocytes specific for HTLV-I pX in patients with HTLV-I associated neurological disease. Nature. 348:245-248.

21. Bieganowska, K., et al. 1999. Direct analysis of viral-specific CD8+ T-cells with soluble HLAA2/Tax11-19 tetramer complexes in patients with HTLV-I-associated myelopathy. J. Immunol. 162:1765-1771.

22. Bieganowska, K., et al. 1997. Direct ex vivo analysis of activated, Fas-sensitive autoreactive T cells in human autoimmune disease. J. Exp. Med. 185:1585-1594.

23. Scholz, C., Patton, K., Freeman, G.J., and Hafler, D.A. 1998. Expansion of autoreactive T cells in multiple sclerosis is independent of exogenous B7 costimulation. J. Immunol. 160:1532-1538 